\title{
EFFECT OF WAVES ON THE BEHAVIOR OF OFFSHORE PILES
}

\author{
Yasser A. El-Hakem and Yehia B. M. Heza \\ Construction Research Institute, National Water Research Center, \\ Cairo, Egypt.
}

\begin{abstract}
This paper studies the effect of wave loads on the submerged piles. The submerged piles are used widely in harbors, and offshore structures. The bottom part of the pile is buried in soil under the mud line while the upper part (free height of the pile above the mud line) is subjected to waves. The internal forces induced in the pile depend on many factors such as, pile diameter, soil type, water depth, wave period and height. A numerical model for a submerged pile was developed using a commercial Finite Element program to study the internal forces induced on submerged piles which subjected to wave action. The factors affecting the internal forces induced in the pile, due to the wave load, were studied. In the present research, range of different values for each factor was taken into consideration to establish the effect of each factor on the internal forces induced in the pile. The results were summarized in curves to highlight the effect of each factor.

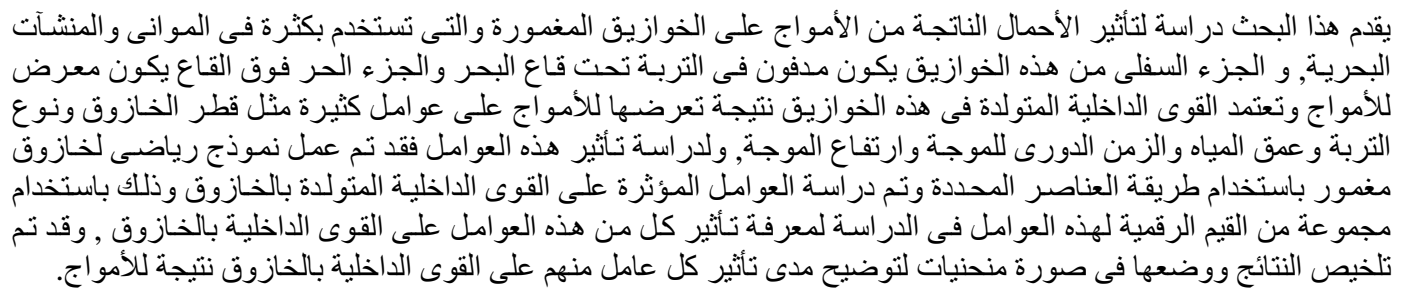

Keywords: Pile, Wind wave, Tidal wave, Drag forces, Offshore, Storm depth, Wave height, Wave celerity, Wave period, and Embedded depth.

\section{INTRODUCTION}

Wind wave generated in the sea area are random and short crested. Most wave theories have been developed for long-crested periodic waves, but they have been applied to waves in the sea and along the coastal zone with satisfactory results. Theoretical treatments of wave motion consider the wave profile to be sinusoidal. The vertical distance between the crest and the following trough is the wave height $\mathrm{H}$, the amplitude " $\mathrm{a}$ " is half of the wave height. The wave length $\mathrm{L}$, is the horizontal distance between two successive crests, while the wave celerity $\mathrm{C}$ is given by $\mathrm{L} / \mathrm{T}$, where $\mathrm{T}$ and $\mathrm{L}$ are the wave period and length, respectively. If the wave height $\mathrm{H}$ has a small value compared to the wave length $\mathrm{L}$ and water depth $\mathrm{d}$, then the governing equations will be linear and the wave form is considered as linear or Airy wave. For large wave heights, nonlinear wave occurs. Stokes waves (deep water, large wave height) and Cnoidal waves (shallow water, long waves) are other waves form that engineers may use as a better approximation to the actual wave records. Both stokes and Cnoidal waves are a symmetrical with respect to the still water level and have sharp crests and elongated troughs. Figure 1, shows the classification of waves related to water depth and the field of application of various wave theories as a function of wave characteristics and water depth d, [1]. Sampiath et al. [2] studied the hydrodynamic loading on offshore structures using computational fluid dynamic. Wave kinematics were used to determine the forces in the pile induced by the wave using Morrison's equation [3]. The wave force is approximated using stokes wave theory which can solve the non linear wave motion [4]. In the present research, numerical models using FEM for different piles with different wave conditions were developed to study the different factors affecting the internal forces induced in the piles. 


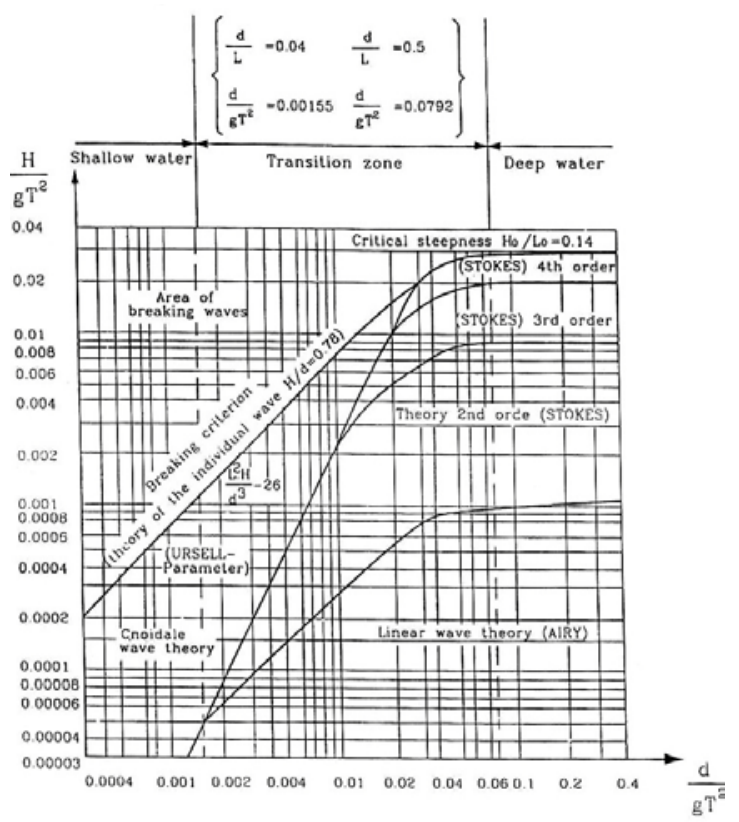

Fig.1 Field of application of various wave theories as a function of wav characteristics and water depth.

\section{GOVERNING EQUATIONS}

For describing the water wave motion, standard assumptions must be introduced such as; the water is inviscid and incompressible, and the flow is irrotational . The governing equations of motion and continuity equation my be written as:

$$
\frac{\partial \phi}{\partial t}+\frac{1}{2}\left[\left(\frac{\partial \phi}{\partial x}\right)^{2}+\left(\frac{\partial \phi}{\partial z}\right)^{2}\right]+\frac{P}{\rho}+g z=0
$$

Where

$\phi$ : the velocity potential given by $\mathrm{u}=\partial \phi / \partial \mathrm{x}$ and $\mathrm{w}$ $=\partial \phi / \partial \mathrm{z}, \mathrm{u}$ and $\mathrm{w}$ are the horizontal and vertical component of the water particle velocity,

P: pressure,

$\rho$ : fluid density,

g: acceleration due to gravity,

$\mathrm{x}$ and $\mathrm{z}$ : horizontal and vertical spatial coordinates and,

t: time.

Laplace equation (Continuity of flow) is given by:

$$
\frac{\partial^{2} \phi}{\partial x^{2}}+\frac{\partial^{2} \phi}{\partial z^{2}}=0
$$

To obtain a solution for the surface wave motion, boundary conditions must be given at the bottom and free surface as well as at the lateral boundaries. The bottom and free surface conditions are usually the same from one case to another, but the lateral boundary conditions vary depending upon the situation (progressive waves, standing wave).

- At the sea bottom, the normal component of water particle velocity is zero, which is given by:

$$
\begin{aligned}
& w=\frac{\partial \phi}{\partial z}=0 \\
& \text { at } z=-d
\end{aligned}
$$

- At free surface $z=\eta$ two boundary conditions are imposed, as follows;

1- The kinematics boundary condition requires that a water particle on the surface remain there.

For which

$$
\frac{\partial \phi}{\partial z}=\frac{\partial \eta}{\partial t} \quad, \quad \text { at } \mathrm{z}=\eta
$$

The dynamic boundary condition requires that the pressure at the surface be equal to the atmospheric pressure $\mathrm{Pa}$, for which

$$
\frac{\partial \phi}{\partial t}+\frac{1}{2}\left[\left(\frac{\partial \phi}{\partial x}\right)^{2}+\left(\frac{\partial \phi}{\partial z}\right)^{2}\right]+\frac{P_{a}}{\rho}+g \eta=0
$$

Neglecting terms involving the square of the velocity and for small amplitude wave, Eq. (1), becomes:

$$
\frac{\partial \phi}{\partial t}+\frac{P}{\rho}+g z=0
$$

substituting for $\mathrm{P}_{\mathrm{a}}=0$ Eq. (5), becomes:

$$
\eta=\frac{1}{g} \frac{\partial \phi}{\partial t} \quad \text { at } \mathrm{z}=0
$$

Eq. (4), may also be written as:

$$
\frac{\partial \phi}{\partial z}=\frac{\partial \eta}{\partial t} \quad \text { at } \mathrm{z} \approx 0
$$

\section{NUMERICAL MODEL}

Finite Element Model for the pile was performed using SAP2000 Nonlinear package [5] using wave loads according to Airy, Stokes, and Cnoidal wave 
theories to model the submerged piles exposed to different waves. Most of the selected values for the different parameters of the waves are choosing according to the field of application of stokes wave theory (as shown in Fig.1). The other wave theories were used in the research to investigate the differences between them.

\subsection{Wave Load}

The program calculates the forces exerted by a wave at a particular location on the pile. The wave loads, shown in Fig. (2), are applied to only the portion of the pile that is above the mud line and below the wave surface. The Morison Equation was applied to calculate the force exerted by the wave. The wave period used when calculating the wave water particle velocities and accelerations, was calculated by solving a system of simultaneous nonlinear equations. These equations are documented in the commentary of the API Recommended Practice (American Petroleum Institute 2000) [6]. Morison equation given by [6] is

$$
F=F_{D}+F_{I}=C_{D} \frac{w}{2 g} A U \mid U+C_{m} \frac{w}{g} V \frac{d U}{d t}
$$

where;

F : Hydrodynamic force per unit length acting normal to the pile longitudinal axis.

F D : Drag force per unit length.

F I : Inertia force per unit length.

C D: Drag coefficient.

$\mathrm{w}$ : Weight density of water.

g : Gravitational acceleration.

A : Projected area normal to pile axis per unit length.

$\mathrm{V}$ : Displaced volume per unit length.

$\mathrm{U}$ : Component of the water particle velocity acting normal to the

axis of the pile.

$|\mathrm{U}|$ : The absolute value of $\mathrm{U}$.

$\mathrm{C} \mathrm{M}$ : Inertia coefficient.

(dU/dt) : Component of the water particle acceleration acting normal to the axis of the pile.

\subsection{Laterally Loaded Pile}

There are many methods for analyzing the response of a laterally loaded pile. These methods can be categorized into subgrade reaction approach and elastic continuum approach. Subgrade reaction approach has been initially proposed by Palmer \& Thompson in 1948, then further advancements lead to the development of p-y curves and are commonly used to model the non-linear pile and soil behaviour. These have been summarized by Reese \& Van Impe (2001) [7]. In the current paper, the subgrade reaction approach is used to analyze the submerged pile (to model the embedded length of pile below the mud line). It characterizes the soil as a series of unconnected linearly-elastic springs in response to loading on the pile. Fig. 3 shows the subgrade reaction model for the pile. In this model, the horizontal pressure (p) and the corresponding deflection at a point (y) is related by a horizontal modulus of subgrade reaction (kh):

$$
\mathrm{p}=\mathrm{kh} \mathrm{y} \text {, }
$$

where:

$\mathrm{p}$ : soil reaction per unit length of pile, and $\mathrm{y}:$ pile deflection,

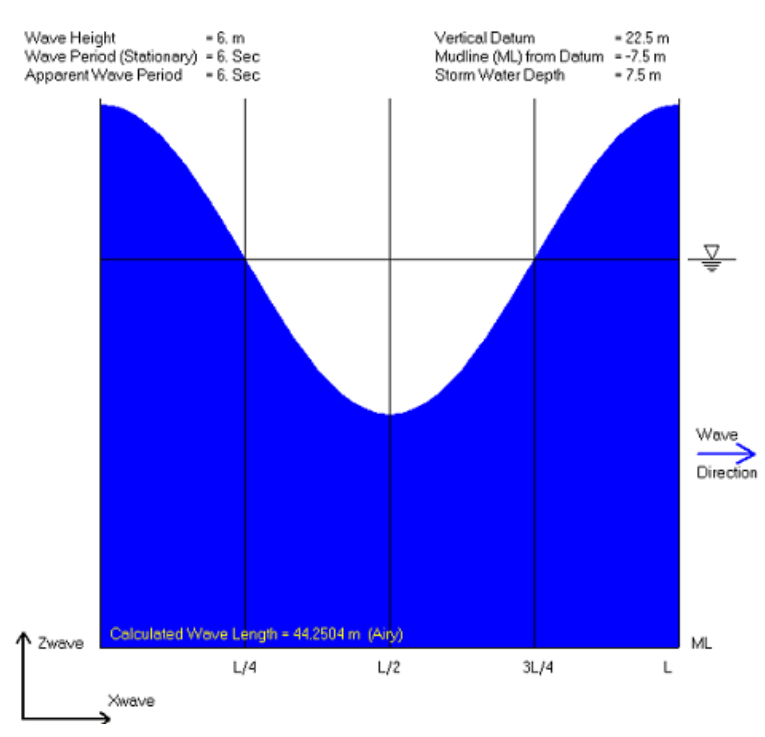

Fig. 2 The Wave Loads used in the Study.

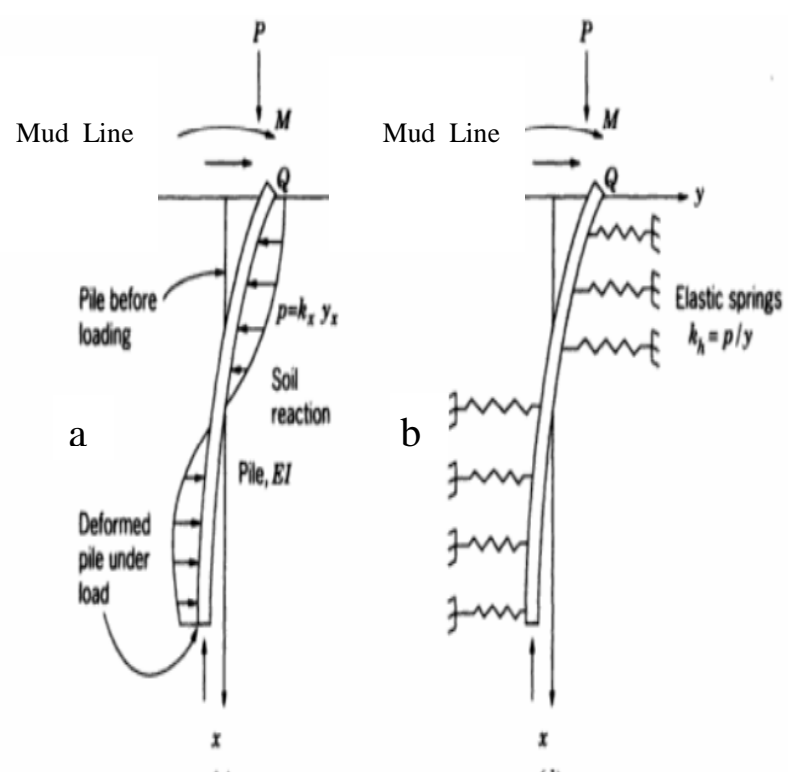

Fig. 3 Subgrade Reaction Model of (a) Actual Soil Reaction on Pile \& (b) Elastic Spring Model of Soil Reaction 
Frame elements were used to model of the pile in the Finite Element model. The soil is modeled as lateral springs with stiffness depend on the lateral subgrade reaction of soil and the area around the spring. Lateral subgrade reaction of soil $=5000$ $\mathrm{t} / \mathrm{m} 3$ was used (to model a pile embedded in clay [8]) to study the effect of the different factors except the soil type. Then lateral springs with different stiffness are used to study the effect of soil type.The free height (the portion of the pile above the mud line to the top of the pile) was increased when the wave height increased to insure that there is a free height above the wave to the top of the pile. For example; the free height of the pile was taken $=$ water depth $+2.5 \mathrm{~m}$ for wave height $=$ $4 \mathrm{~m}$ to get $0.5 \mathrm{~m}$ free board.

\section{EFFECT OF WAVE HEIGHT}

To study the effect of wave height on the internal forces induced in the pile, a pile with diameter = $0.5 \mathrm{~m}$ and with free height $=15 \mathrm{~m}$, corresponding to water depth $=12.5 \mathrm{~m}$, was taken in the research. The wave period was taken 6 seconds, and the embedded depth was taken $15 \mathrm{~m}$. The wave height of the pile was taken $2 \mathrm{~m}, 4 \mathrm{~m}, 6 \mathrm{~m}$ and $8 \mathrm{~m}$. Figure 4 , shows the relation between the maximum moment induced in the pile and the wave height.

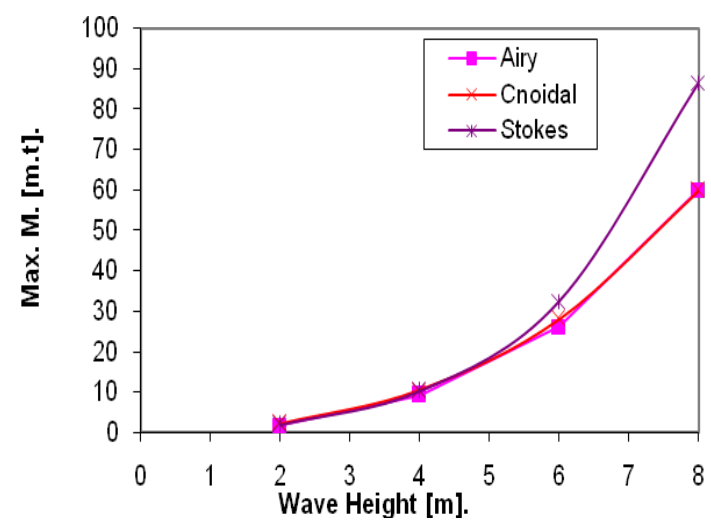

Fig. 4 the Relation between Max. Moment and Wave Height for $12.5 \mathrm{~m}$ Water Depth

As shown in Fig. 4, when the wave height increases from $2 \mathrm{~m}$ to $8 \mathrm{~m}$, the maximum moment increases (by about 30\% when using Airy and Cnoidal wave theories to $45 \%$ when using Stokes wave theory). The rate of increasing in the moment increases exponentially. Stokes wave theory gives higher results than Airy and Cnoidal wave theories especially when the wave height increases and reaches $8 \mathrm{~m}$, (near the area of the breaking wave which is shown in Fig.1.

\section{EFFECT OF WATER DEPTH}

Studying the effect of water depth on the internal forces induced in the pile, the water depth was taken $7.5 \mathrm{~m}, 12.5 \mathrm{~m}, 17.5 \mathrm{~m}$ and

22.5m (corresponding to a pile with free height $10 \mathrm{~m}, 15 \mathrm{~m}, 20 \mathrm{~m}$ and $25 \mathrm{~m}$, respectively). The wave period, the wave height, and the pile diameter were taken 6 seconds, $4 \mathrm{~m}$, and $0.5 \mathrm{~m}$, respectively. Figure 5 , shows the relation between the maximum moment induced in the pile and the water depth. As shown in Fig. 5, the maximum moment induced in the pile increases by about 25\% during increasing water depth from $7.5 \mathrm{~m}$ to $17.5 \mathrm{~m}$, using Stokes and Cnoidal wave theory. This relation is linear when using airy wave theory. Also the figure shows that, Cnoidal and Stokes wave theories give higher results compared with using Airy wave theory especially when the water depth decreases. The moment by using Cnoidal and Stokes was about 48\%, and 3\% higher than Airy when water depth was $7.5 \mathrm{~m}$, and $17.5 \mathrm{~m}$, respectively.

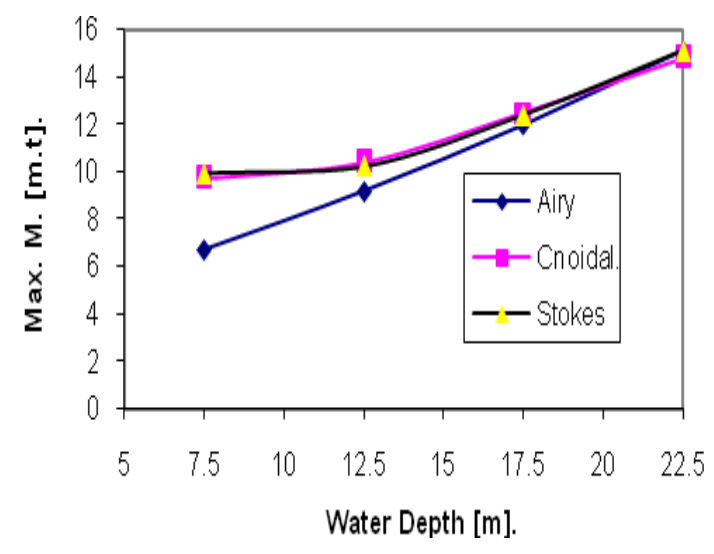

Fig. 5 the Relation between Maximum Moment and Water Depth

When water depth reaches $22.5 \mathrm{~m}$, the results were approximately the same. This means that the difference in the results among the three wave theories decreases when the water depth increases.

\section{EFFECT OF WAVE PERIOD}

Considering the effect of wave period on the internal forces induced in the pile, the pile diameter was taken $0.5 \mathrm{~m}$, and the free height was taken 10 , 15 and $20 \mathrm{~m}$ corresponding to water depth $=7.5$, $12.5,17.5 \mathrm{~m}$, respectively. The wave period was taken 4, 6 and 8 seconds, the embedded depth of the pile and the wave height were taken $15 \mathrm{~m}$ and 4 $\mathrm{m}$, respectively. Figure 6, shows the relation between the maximum moment induced in the pile and the wave period for the different pile free 
heights and water depths. As shown in Fig. 6, considering Airy and Stokes wave theories, with a wave period increases from 4 to 6 seconds, the maximum moment decreases, (from about $29 \%$ at water depth $=7.5 \mathrm{~m}$ to $38 \%$ at water depth $=17.5 \mathrm{~m}$ when using Stokes and from about $13 \%$ at water depth $=7.5 \mathrm{~m}$ to $34 \%$ at water depth $=17.5 \mathrm{~m}$ when using Airy). Using Cnoidal wave theory, with increasing the wave period increases, the maximum moment increases. When the wave period increased from 6 to 8 seconds the rate of change in the moment became low (from about $1 \%$ to $10 \%$ ).
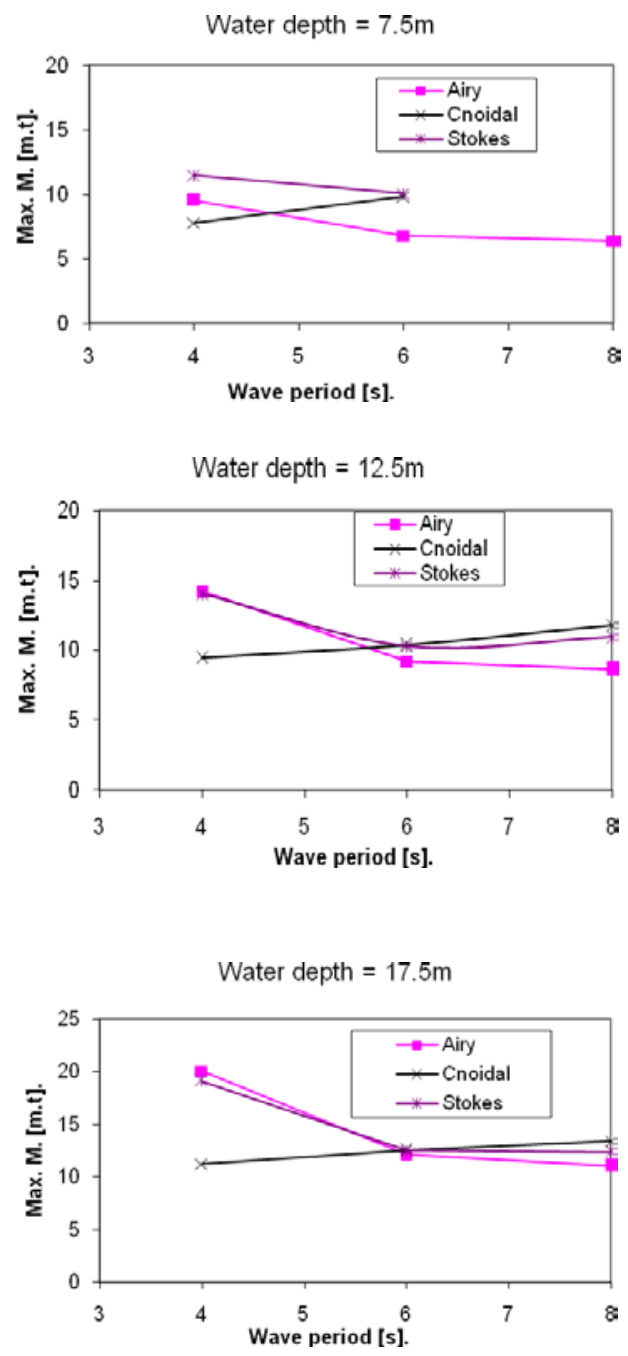

Fig. 6 the Relation between Maximum Moment and Wave Period

\section{EFFECT OF PILE DIAMETER}

Regarding to the effect of pile diameter on the internal forces induced in the pile, the water depth was taken $7.5 \mathrm{~m}, 12.5 \mathrm{~m}, 17.5 \mathrm{~m}$ and $22.5 \mathrm{~m}$. The wave height, the wave period and the embedded depth of the pile were taken $4 \mathrm{~m}, 6$ seconds and $15 \mathrm{~m}$, respectively. The pile diameter was taken $0.5 \mathrm{~m}, 1 \mathrm{~m}, 5 \mathrm{~m}$ and $8 \mathrm{~m}$. Figure 7 , shows the relation between the maximum moment induced in the pile and the pile diameter. As shown in Fig. 7, when the pile diameter increases the moment increases the relation is approximately linear in all theories. The moment increased from 10 m.t when the pile diameter was $0.5 \mathrm{~m}$ to $183 \mathrm{~m}$.t with pile diameter 8 $\mathrm{m}$.
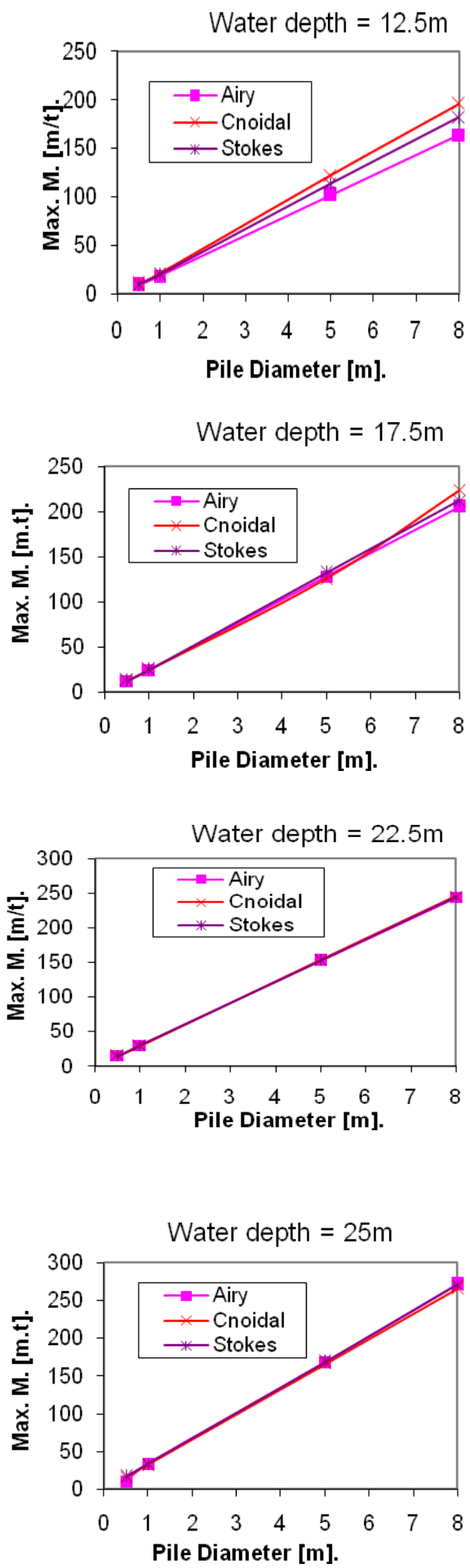

Fig. 7 the Relation between Maximum Moment and Pile Diameter 
This results happened because the pile which has a great diameter results in a great area exposed to the wave. Also, Fig. 7 shows that when the water depth increases the results of the three wave theories become approximately the same.

\section{EFFECT OF SOIL TYPE}

As for the effect of soil type on the internal forces induced in the pile, a pile with diameter $=0.5 \mathrm{~m}$ was studied, the water depth, the wave period, the wave height, and the embedded depth were taken $12.5 \mathrm{~m}$, 6 seconds, $4 \mathrm{~m}$, and $15 \mathrm{~m}$, respectively.

The commonly used form and adopting a variation of kh with depth [9]:

$\mathrm{k} \mathrm{h}=\mathrm{n} \mathrm{h}(\mathrm{z} / \mathrm{d})$,

Where nh is the coefficient of subgrade reaction and $\mathrm{d}$ is pile diameter. In Eq. (11) the values of $\mathrm{nh}$ were taken 139, 485 and $1178 \mathrm{t} / \mathrm{m} 3$ for loose, medium, and dense submerged sand, respectively [9]. This applies to cohesionless soils which indicate increasing strength with depth due to the increase in the overburden pressure. The cohesive were also studied where the lateral subgrade reaction is constant with depth. For cohesive soil with Kh does not increase with depth, the values of the lateral subgrade reaction were taken 2087, 3544 , and $5000 \mathrm{t} / \mathrm{m} 3$ for soft, soft to medium and medium clay, respectively [8]. The depth at zero moment under the mud line is very important to the cost of piles because it controls the length of the pile (the pile length decreases when this depth decreases). Fig. 8 shows the relation between the lateral subgrade reactions of soil and the depth at zero moment under the mud line. The average values of the lateral subgrade reactions of the cohesionless soil were used when plotting this figure because these values are variable (increase with the depth) for the same soil.

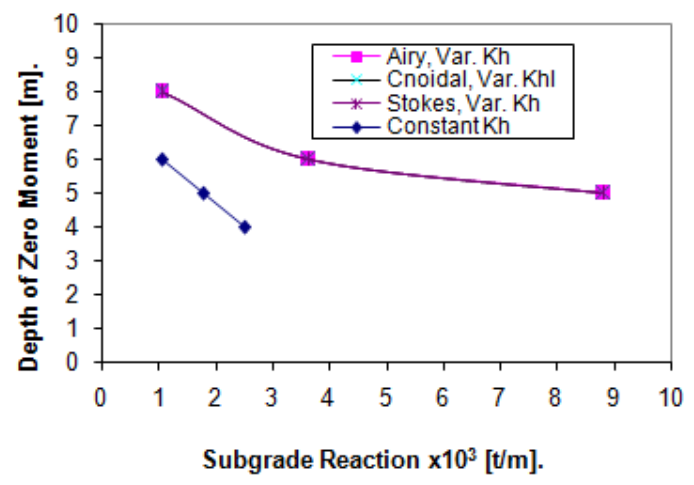

Fig. 8 the Relation between Soil Lateral Subgrade Reaction and Depth of Zero Moment.
The depth at zero moment was the same in the three wave theory. This depth decreases (by about $33 \%$ to $38 \%$ ) when the lateral subgrade reaction of the soil increases. These depths are higher in cohesionless soil than that in cohesive soil by about (from 25\% to 33\%). This is because the values of the subgrade reactions are small near the mud line in cohesionless soil.

\section{CONCLUSIONS}

The results obtained from the numerical model leads to the following conclusions:

The wave height has a great effect on the results. The maximum moment increases when the wave height increases. The rate of increasing in the moment increases exponentially.

The maximum moment induced in the pile increases when the water depth increases. This relation is linear when using airy wave theory. Cnoidal and Stokes wave theories give higher results than that when using Airy wave theory. The difference in the results among the three wave theories decreases when the water depth increases.

Using Airy and Stokes wave theories, when the wave period increases the maximum moment decreases. By using Cnoidal wave theory, when the wave period increases, the maximum moment increases. The rate of change in the moment became low in the long wave periods. When the pile diameter increases the moment increases. The relation is approximately linear in the three wave theories. This moment reaches very big values in piles with great diameters. The depth at zero moment (which affects the length of the pile and its cost) decreases (by about 33\% to 38\%) when the lateral subgrade reaction of the soil increases. These depths are higher in cohesionless soil than that in cohesive soil.

\section{REFERENCES}

[1] Holmes, P.," Coastal Processes: Waves", A Course in Coastal Defense Systems, Organized by Civil Engineering Dept., The University of the West Indies, in conjunction with Old Dominion University, USA and Coastal Engineering Research Centre, US Army, Corps of Engineers, Vicksburg, MS, USA. St. Lucia, West Indies, July 18-21, 2001.

[2] Sampiath,A., Allan, M., and Kostas, L., "CFD as a Design Tool for Hydro Dynamic Loading on Offshore Structures", Prooceeding of ASME 28th International Conference on Ocean, Offshore and Arctic Engineering Hawai, USA, 2009.

[3] Navarre Beach Fishing Pier Design Report, "Wave Load Calculations, Methodology \& Results", Coastal and Water Ways, Division, PBS\&J, Corporation, USA, July,2007. 
[4] Son, C. Y., Lee, K., Lee, J., and Kim, K.," A Study on the Sensitivity of Dynamic Behavior of Jacket Type Offshore Structures", INHA University, Korea, 7th World Wind Energy: Energy Autonomy and Local Economies St Lawrence College in Kingston Ontario, Toronto, Canada, 2008.

[5] Computers and Structures, Inc., "Sap2000 Nonlinear Ver. 10 Reference Manual”, Berkeley, California, USA, July 2005.

[6] "-Recommended Practice for Planning, Designing and Constructing Fixed OffshorePlatforms-Working Stress Design", American Petroleum Institute (API), Recommended Practice, Twinty-First Edition, December, 2000.
[7] Reese, L.C. \& Van Impe, W.F., "Single Piles \& Groups under Lateral Loading", A.A. Balkema, UK, 2001.

[8] Bowles, J., et. al, "Foundation Analysis and Design", Fifth Edition, The McGraw-Hill Companies, Inc., 1996.

[9] Palmer, L.A. \& Thompson, J.B. (1948). The Earth Pressure and Deflection Along theEmbedded Lengths of Piles Subjected to Lateral Thrust. Proceedings of the Second International Conference on Soil Mechanics and Foundation Engineering, Rotterdam, Vol. 5, pp 156-161. 